Pacific Journal of Mathematics

SKEW LINEAR VECTOR FIELDS ON SPHERES IN THE 


\title{
SKEW LINEAR VECTOR FIELDS ON SPHERES IN THE STABLE RANGE
}

\section{J. C. BECKER}

\begin{abstract}
Theorem. Assume $n>2 k$. Then every $(k-1)$-field on $S^{n-1}$ is skew linear.
\end{abstract}

1. Introduction. Skew linear vector fields on spheres have been studied by Strutt [6], Zvengrowski [8] and Milgram and Zvengrowski $[4,5]$. Extensive calculation of projective homotopy classes in [5] led Milgram and Zvengrowski to conjecture that every $r$-field on $S^{n-1}$ is skew linear. Here we will prove this conjecture in the stable range, as stated above.

After a reformulation using a construction of L. Woodward [7] and the results of [1], the theorem will follow from the Kahn-Priddy theorem [3].

Since proving this theorem I have learned that Milgram and Zvengrowski had already obtained the result using different methods [9]. They have also shown that 7 and 8-fields on $S^{15}$ are skew linear, the two remaining cases excluded by the condition $n>2 k$ and not already dealt with in [8]. L. Woodward has also proved the theorem by methods similar to those used here.

2. Proof of the theorem. If $p: E \rightarrow B$ is a fibration let $C(B ; E)$ denote the set of vertical homotopy classes of cross sections to $p$. If $Z_{2}$ acts freely on $B$ and $E$ in such a way that $p$ is equivariant let $C_{Z_{2}}(B ; E)$ denote the set of equivariant vertical homotopy classes of equivariant cross sections to $p$.

Let $V_{n, k}$ denote the Stiefel manifold of $k$-frames in $R^{n}$ with the involution $\left[v_{1}, \cdots, v_{k}\right] \rightarrow\left[-v_{1}, \cdots,-v_{k}\right]$. Recall that a skew linear $(k-1)$-field on $S^{n-1}$ is a cross section to the bundle $V_{n, k} \rightarrow S^{n-1}$ which is vertically homotopic to an equivariant cross section. Let $L_{n, k}$ denote the space of equivariant maps $S^{k-1} \rightarrow S^{n-1}$. Fixing $x_{0}=(1,0, \cdots, 0) \in S^{k-1}$ as base point we have a fibration $L_{n, k} \rightarrow S^{n-1}$ by evaluating at $x_{0}$ and a commutative square

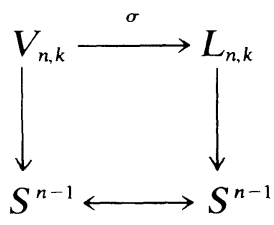


where $\sigma$ is the natural inclusion. The antipodal map on $S^{n-1}$ induces an involution on $L_{n, k}$ such that the maps in the above diagram are equivariant. As is well known [2], $\sigma$ is a $(2(n-k)-1)$ equivalence. Hence

$$
C\left(S^{n-1} ; V_{n, k}\right) \simeq C\left(S^{n-1} ; L_{n, k}\right)
$$

and

$$
C_{Z_{2}}\left(S^{n-1} ; V_{n, k}\right) \simeq C_{Z_{2}}\left(S^{n-1} ; L_{n, k}\right) .
$$

Let $P_{k}$ denote $(k-1)$-dimensional real projective space and $\eta_{k}$ the Hopf bundle over $P_{k}$. Let $\operatorname{Tr}\left(n \eta_{k}\right)$ (respectively, $\operatorname{Tr}_{Z_{2}}\left(n \eta_{k}\right)$ ) denote the set of fiber homotopy classes of fiber preserving maps (respectively, equivariant fiber homotopy classes of equivariant fiber preserving maps) $P_{k} \times S^{n-1} \rightarrow S\left(n \eta_{k}\right)$, whose restriction to the fiber over $\left[x_{0}\right]$ is the identity map. Here $S\left(n \eta_{k}\right)$ is the unit sphere bundle of $n \eta_{k}$. Define a map

$$
\mu: C\left(S^{n-1} ; L_{n, k}\right) \rightarrow \operatorname{Tr}\left(n \eta_{k}\right)
$$

by $\Delta \rightarrow \tilde{\Delta}$ where $\tilde{\Delta}([x], y)=[x, \Delta(y)(x)], x \in S^{k-1}, y \in S^{n-1}$. This map is a bijection; in fact the underlying function spaces are homeomorphic (see Woodward [7, Lemma 1,2]). Similarly we have a bijection

$$
\mu_{Z_{2}}: C_{Z_{2}}\left(S^{n-1} ; L_{n, k}\right) \rightarrow \operatorname{Tr}_{Z_{2}}\left(n \eta_{k}\right)
$$

Let $G\left(S^{n-1}\right)$ denote the identity component of the space of maps $S^{n-1} \rightarrow S^{n-1}$ and let $G=$ inj. $\lim . G\left(S^{n-1}\right)$. Let $G_{Z_{2}}=$ inj. $\lim . G_{Z_{2}}\left(S^{n-1}\right)$ where $G_{Z_{2}}\left(S^{n-1}\right)$ is the identity component of the space of equivariant maps $S^{n-1} \rightarrow S^{n-1}$. Fixing an equivariant fiber map $f: S\left(n \eta_{k}\right) \rightarrow P_{k} \times$ $S^{n-1}$ whose restriction to the fiber over $\left[x_{0}\right]$ is the identity, we have equivalences

$$
\nu: \operatorname{Tr}\left(n \eta_{k}\right) \rightarrow\left[P_{k} ; G\right]
$$

and

$$
\nu_{Z_{2}}: \operatorname{Tr}_{Z_{2}}\left(n \eta_{k}\right) \rightarrow\left[P_{k} ; G_{Z_{2}}\right]
$$

Each of these is defined by sending $h: P_{k} \times S^{n-1} \rightarrow S\left(n \eta_{k}\right)$ to the adjoint of

$$
P_{k} \times S^{n-1} \stackrel{h}{\rightarrow} S\left(n \eta_{k}\right) \stackrel{f}{\rightarrow} P_{k} \times S^{n-1} \rightarrow S^{n-1}
$$


Here [;] denotes homotopy classes of base point preserving maps.

Summarizing, let

$$
\psi: C\left(S^{n-1} ; V_{n, k}\right) \rightarrow\left[P_{k} ; G\right]
$$

denote the composite

$$
C\left(S^{n-1} ; V_{n, k}\right) \stackrel{\sigma *}{\rightarrow} C\left(S^{n-1} ; L_{n, k}\right) \stackrel{\mu}{\rightarrow} \operatorname{Tr}\left(n \eta_{k}\right) \stackrel{\nu}{\rightarrow}\left[P_{k} ; G\right]
$$

and let $\psi_{z_{2}}$ denote its equivariant analogue.

Lemma. Assume $n>2 k$. There is a commutative square

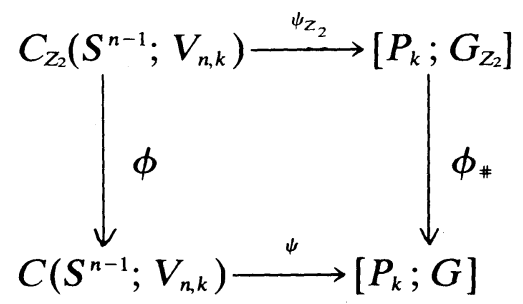

in which $\psi$ and $\psi_{Z_{2}}$ are equivalences and $\phi$ is the forgetful map.

If $X$ is a connected space let $Q^{0}\left(X^{+}\right)$denote the $o$-component of $Q\left(X^{+}\right)=\Omega^{\infty} S^{\infty}\left(X^{+}\right)$. By the main result of [1] there is a commutative square

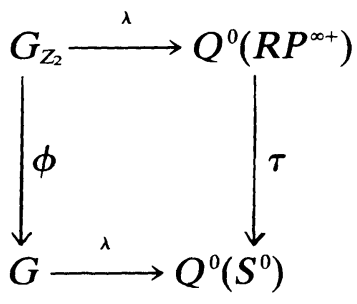

in which the horizontal maps are homotopy equivalences and $\tau$ is the transfer map associated with the double cover $S^{\infty} \rightarrow R P^{\infty}$. In view of this and the above lemma, our theorem will follow by showing that

$$
\tau_{\#}:\left[P_{k} ; Q^{0}\left(R P^{\infty+}\right)\right] \rightarrow\left[P_{k} ; Q^{0}\left(S^{0}\right)\right]
$$

is epimorphic. This is a consequence of the Kahn-Priddy theorem [3]. First note that both of these groups are finite and $\tau_{*}$ is clearly onto 
the odd primary part. The Kahn-Priddy result states that $\tau_{\#}$ also maps onto the 2-primary part. (Although they only consider the morphisms $\tau_{\#}:\left[S^{m} ; Q^{0}\left(R P^{\infty+}\right)\right] \rightarrow\left[S^{m} ; Q^{0}\left(S^{0}\right)\right]$, for all $m$, their proof is valid with $S^{m}$ replaced by any finite complex.)

\section{REFERENCES}

1. J. C. Becker and R. E. Schultz, Equivariant function spaces and stable homotopy theory I, Comm. Math. Helv., 49 (1974), 1-34.

2. A. Haefliger and M. W. Hirsch, Immersions in the stable range, Ann. Math., 75 (1962), 231-241.

3. D. S. Kahn and S. B. Priddy, Applications of the transfer to stable homotopy theory, Bull. Amer. Math. Soc., 78 (1972), 981-987.

4. R. J. Milgram and P. Zvengrowski, Projective Stiefel manifolds and skew linear vector fields, Proc. London Math. Soc., 28 (1974), 671-682.

5. Stable projective homotopy and applications to skew linear vector fields, to appear.

6. J. Strutt, Projective homotopy of Stiefel manifolds, Canad. J. Math., 24 (1972), 465-476.

7. L. M. Woodward, Vector fields on spheres and a generalization, Quart. J. Math., 24 (1973), 357-366.

8. P. Zvengrowski, Skew linear vector fields on spheres, J. London Math. Soc., 3 (1971), 625-632.

9. R. J. Milgram and P. Zvengrowski, Skew linearity of r-fields on spheres, Topology, to appear.

Received February 3, 1976. Research supported by the Science Research Council (Gt. Britain) and the National Science Foundation.

Purdue University 



\section{Pacific Journal of Mathematics}

Vol. 68, No. 1

March, 1977

Richard Julian Bagby, On $L^{p}, L^{q}$ multipliers of Fourier transforms . .......

Robert Beauwens and Jean-Jacques Van Binnebeek, Convergence theorems in

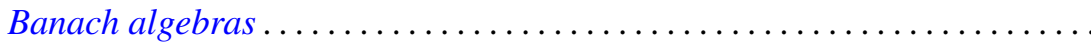

James Cyril Becker, Skew linear vector fields on spheres in the stable

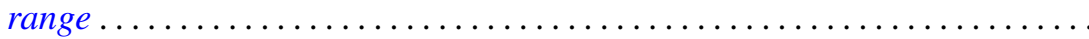

Michael James Beeson, Continuity and comprehension in intuitionistic formal

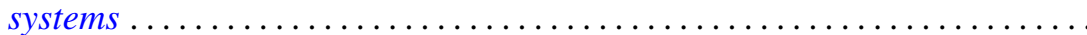

James K. Deveney, Generalized primitive elements for transcendental field

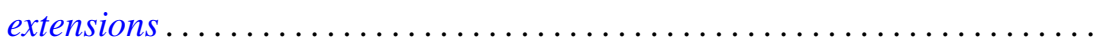

Samuel S. Feder, Samuel Carlos Gitler and K. Y. Lam, Composition properties

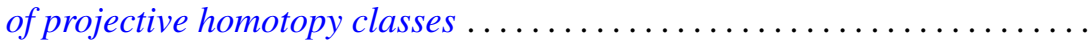

Nathan Jacob Fine, Tensor products of function rings under composition ......

Benno Fuchssteiner, Iterations and fixpoints . . . . . . . . . . . . . .

Wolfgang H. Heil, On punctured balls in manifolds

Shigeru Itoh, A random fixed point theorem for a multivalued contraction

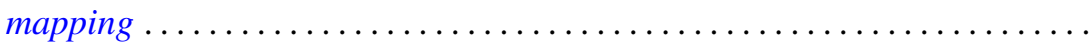

Nicolas P. Jewell, Continuity of module and higher derivations . . . . . . ......

Roger Dale Konyndyk, Residually central wreath products . . . . . . . . . . .

Linda M. Lesniak and John A. Roberts, On Ramsey theory and graphical

parameters.

Vo Thanh Liem, Some cellular subsets of the spheres.

Dieter Lutz, A perturbation theorem for spectral operators

P. H. Maserick, Moments of measures on convex bodies ... . . .

Stephen Joseph McAdam, Unmixed 2-dimensional local domains . .

D. B. McAlister and Norman R. Reilly, E-unitary covers for inverse semigroups...

William H. Meeks, III and Julie Patrusky, Representing codimension-one

homology classes by embedded submanifolds . . .

Premalata Mohapatro, Generalised quasi-Nörlund summability . .

Takahiko Nakazi, Superalgebras of weak-*Dirichlet algebras .

Catherine Louise Olsen, Norms of compact perturbations of operators .

William Henry Ruckle, Absolutely divergent series and isomorphism of

subspaces. II.

Bernard Russo, On the Hausdorff-Young theorem for integral operators .

Arthur Argyle Sagle and J. R. Schumi, Anti-commutative algebras and

homogeneous spaces with multiplications ............

Robert Evert Stong, Stiefel-Whitney classes of manifolds .

D. Suryanarayana, On a theorem of Apostol concerning Möbius functions of

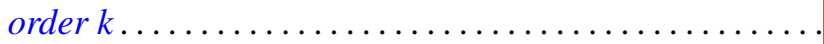

Yoshio Tanaka, On closedness of $C$ - and $C^{*}$-embeddings . . 